\title{
Womenomics en Japón: Mujer, neoliberalismo y paradigma productivista
}

\author{
Womenomics in Japan: Women, Neoliberalism \\ and Productivist Paradigm
}

MONTSERRAT CRESPÍN PERALES (Universitat de Barcelona; Universitat Autònoma de Barcelona)

Artículo recibido: 31 de julio de 2018

Solicitud de revisión: 21 de octubre de 2018

Artículo aceptado: 9 de enero de 2019

Crespín Perales, Montserrat (2019). Womenomics en Japón: Mujer, neoliberalismo y paradigma productivista. Recerca. Revista de Pensament i Análisi, 24(2), pp. 63-86.

\section{Resumen}

El concepto womenomics propone la idea de «comprar la economía femenina», designando con ello la necesidad de Japón de hacer esfuerzos por incluir exponencialmente a la mujer en el mercado laboral e introducir uno de los mecanismos para corregir un futuro de estancamiento y contracción del crecimiento económico. El concepto lo acuñó en 1999 Kathy M. Matsui al frente de la división de investigación en Asia de economía, materias primas y estrategia del grupo de banca y valores más importantes del mundo, Goldman Sachs. En este ensayo se propone estudiar y analizar críticamente el marco político y económico de la estrategia conocida como Abe-Womenomics con el objetivo de conocer un aspecto de la actual política japonesa doméstica y, por extensión, las implicaciones regionales e internacionales de su adopción y adaptación para, luego, averiguar si este tipo de medidas buscan un giro hacia la equidad genuina o solamente replican un patrón productivista, resignificando las aspiraciones feministas en beneficio del sistema neoliberal.

Palabras clave: Abe-Womenomics, Womenomics, Kathy M. Matsui, Nancy Fraser, neoliberalismo.

Abstract

The concept womenomics proposes the idea of «buying the female economy», thus designating Japan's need to make efforts to exponentially include women in the labor market and introducing one of the mechanisms to correct a future of stagnation and contraction of the economic growth. The concept was coined by Kathy M. Matsui in 1999 at the head of the Asian research division of Economy, Commodities and Strategy of the world's leading banking and investment group, Goldman Sachs. This essay proposes to study and critically ana- 
lyze the political and economic framework of the strategy known as Abe-Womenomics, with the aim of knowing an aspect of current Japanese domestic policy and, by extension, the regional and international implications of its adoption and adaptation. Then, the objective is to demonstrate whether this type of measure seeks a turn towards a genuine equity or only replicates a productivist pattern, re-signifying feminist aspirations for the benefit of the neoliberal system.

Key Words: Abe-Womenomics, Womenomics, Kathy M. Matsui, Nancy Fraser, neoliberalism.

\section{INTRODUCCIÓN}

En un reciente artículo, la especialista en movimientos políticos feministas en Japón, Vera Mackie (2016), advertía de la importancia de estudiar, analizar e interpretar, junto a la política y las relaciones internacionales, la adopción del bloque de medidas políticas conocidas como womenomics por parte del actual primer ministro japonés Shinzō Abe:

Las políticas sobre las relaciones de género deben ser vistas a través del marco transnacional y regional. [...] Tres años después del discurso de Abe sobre el «womenomics», el defectuoso acuerdo bilateral Japón-Corea del Sur ${ }^{1}$ revela la necesidad de una mayor integración a través de las perspectivas feministas no solamente en la política japonesa doméstica sino también en sus relaciones internacionales (Mackie, 2016). ${ }^{2}$

Este ensayo se propone confirmar y justificar la necesidad de la integración de las teorías políticas feministas en su doble eje, a saber, aprendiendo del caso de estudio que remitirá a la adopción del womenomics en la política japonesa nacional y, por extensión, en las implicaciones regionales e internacionales de su adopción y adaptación. El análisis busca abrir un camino posible para la reflexión compartida que, necesitando describir e identificar lo que de singular haya en el caso japonés, sirva específicamente para dar con los mecanismos compartidos y conmensurables.

El concepto womenomics propone la idea de «comprar la economía femenina». Designa la necesidad de Japón de hacer esfuerzos para aumentar expo-

1 Se refiere al acuerdo bilateral firmado en el 2015 entre los gobiernos de Park Geun Hye y Shinzō Abe para clausurar la cuestión sobre las llamadas mujeres de confort (esclavas sexuales, sin eufemismos) coreanas durante la segunda guerra mundial o, en la región, la guerra del Pacífico. El acuerdo, admitiendo el reconocimiento y la indemnización de estas mujeres, tenía por objetivo cerrar una herida que ha mantenido tensionada la relación entre los dos países durante décadas.

2 Con el fin de mantener la coherencia estilística y terminológica del artículo, las traducciones de textos escritos en lenguas distintas a la castellana son mías. 
nencialmente la inclusión de la mujer en el mercado laboral y, con ello, potenciar uno de los mecanismos que se considera puede ayudar a corregir un presente y futuro de estancamiento y contracción del crecimiento económico que, según el pensamiento económico de corte liberal, se sustenta en trabajo, capital y productividad. El término lo acunó Kathy M. Matsui en 1999 en el documento estratégico de Goldman Sachs titulado «Womenomics: compre la economía femenina» (Matsui, Suzuki, Tatebe y Akiba, 2014: 3). Matsui está hoy en día al frente de la división de investigación en Asia de economía, materias primas y estrategia de este grupo de banca y valores, uno de los más importantes del mundo. El concepto y su predicamento han sido sumamente exitosos, como se puede comprobar solamente atendiendo a la repercusión de su teorización en diversos foros económicos de referencia, como, por ejemplo, The Economist (2006).

Para perseguir el propósito último de este ensayo, en primer lugar, se repasarán aspectos que ayuden a situar la importancia de la teoría política feminista en nuestra contemporaneidad y se expondrá hasta qué punto ciertas líneas feministas (no el feminismo entendido erróneamente como monolítico singular) se han trastocado en discursos aliados del neoliberalismo. Luego, se describirá el origen y la significación del concepto político-económico womenomics en el Japón actual, paso ineludible para, posteriormente, interpretar si las medidas del gobierno japonés, conocidas como Abe-Womenomics, buscan un giro hacia la equidad genuina o solamente replican un patrón productivista.

\section{LOS PROCESOS DE RESIGNIFICACIÓN EN EL AUGE DEL NEOLIBERALISMO ECONÓMICO Y POLÍTICO}

Nancy Fraser (2009), en su artículo «El feminismo, el capitalismo y la astucia de la historia», realiza un notable análisis de la segunda ola feminista. Su estudio servirá, más adelante, para conectar la significación del movimiento, la paradójica coincidencia con el neoliberalismo y, por tanto, cuál es el contexto específico que ayuda a entender la lógica tras la implementación actual de la estrategia económica, política y social del proyecto de womenomics en Japón y en el ámbito regional e internacional.

Fraser comienza indicando cuál es su propósito al analizar retrospectivamente la segunda ola feminista: quiere evaluar la trayectoria y la importancia del movimiento y, reconstruyendo ese trayecto, iluminar y clarificar el momento de crisis económica, incertidumbre social y realineamiento político 
(Fraser, 2009: 87). Lo que interesará aquí del enfoque de la pensadora será evidenciar si puede servir como herramienta crítica con respecto a la estrategia de la Abe-Womenomics para establecer si también allí se correlaciona la historia de esa segunda ola del feminismo con la historia del capitalismo contemporáneo. Y, en particular, si es el caso que el Abe-Womenomics es una ilustración de lo que la filósofa indica como «inquietante posibilidad»:

[...] los cambios culturales propulsados por la segunda ola, saludables en sí mismos, han servido para legitimar una transformación estructural de la sociedad capitalista que avanza directamente en contra de las visiones feministas de una sociedad justa (Fraser, 2009: 87).

El primer punto que conviene subrayar remite al proceso de desarrollo y maduración de la segunda ola del feminismo en el interior de lo que Fraser define como «capitalismo organizado de Estado» a partir de 1945. Esto es, la formación social en la que los Estados regulaban sus economías nacionales en el sentido de intervenir y dirigir la inversión, la industria, la tributación, la provisión social o el marco empresarial (Fraser, 2009: 87). Según Fraser, el capitalismo estatal en las décadas posteriores a 1945 se define por su economicismo, el androcentrismo, el estatismo y el westfalianismo. Para la filósofa, el economicismo sería el uso del poder político público para regular los mercados a través de los mecanismos de justicia distributiva sustentados en la renta y el trabajo. El androcentrismo, conceptuaría la idea del ciudadano productivo, equivalente al trabajador varón, de la mayoría étnica social y sostén de la familia nuclear, siendo el trabajo asalariado de la mujer (o esposa) puramente complementario y el no asalariado, de cuidado de la familia o respecto a la incidencia en la mujer de la reproducción, desvalorizado. El estatismo comprende la organización social sustentada en la forma moderna de estado, pero, en particular, de la tecnocracia, es decir, la gestión política en manos de una minoría política burocrática y la consiguiente despolitización o desafección de los ciudadanos. Y, por último, el westfalianismo refiere a la institucionalización del principio según el cual las obligaciones de justicia son aplicables entre conciudadanos, esto es, en el interior de la política interna de los estados (Fraser, 2009: 90-92).

Acto seguido, Fraser indica cómo el feminismo de la segunda ola hizo frente al marco del capitalismo organizado de Estado. Primeramente, sustituyendo la visión economicista por la tridimensional: economía, cultura, política. El feminismo llamó la atención sobre la politización de lo privado, lo 
personal, mostrando que las asimetrías, desigualdades e injusticias responden a diversos parámetros (familiares, culturales, cotidianos, sexuales, reproductivos, etc.). Se criticó también al androcentrismo revelando la importancia de las tareas de las mujeres en áreas no remuneradas (cuidado de la familia y sus miembros), las relaciones de poder y subordinación en el matrimonio y el diseño de las redes de distribución (como prestaciones sociales, organización del trabajo y la industria, etc.). Se trató de llamar la atención sobre la importancia de las mujeres al desempeñar actividades no asalariadas. Se intentó romper con el estatismo promoviendo la participación de las mujeres en la gestión política. Se revisó el marco westfaliano, intentando no caer en el imperialismo intervencionista. En síntesis:

[...] la segunda ola feminista asumió un proyecto político transformador, basado en una interpretación más amplia de la injusticia y en la crítica sistémica a la sociedad capitalista. Las corrientes más avanzadas del movimiento consideraron que las suyas eran unas luchas multidimensionales, dirigidas simultáneamente contra la explotación económica, la jerarquía del estatus y el sometimiento político (Fraser, 2009: 95).

Expuestos los dos movimientos, ¿en qué punto se produce la inquietante convergencia entre la segunda ola feminista y el neoliberalismo? O, como pregunta Fraser, ¿cuándo se produce que el neoliberalismo pujante «resignificara» el feminismo de la segunda ola y sus ideales y aspiraciones? Así pues, se trata de contrastar la hipótesis que sostiene la filósofa respecto al proceso de resignificación (Butler, 1990), por tanto, de la adquisición de un nuevo sentido o sentidos diferentes de los ideales feministas originarios. Es decir, ver en qué términos el neoliberalismo se apropió de ciertas aspiraciones de esa segunda ola feminista dándoles otro significado y valor, acorde con los propios credos del pensamiento político y económico neoliberal. Para Fraser, la resignificación se produce a la vez que la debilitación del antieconomicismo feminista que favorecerá un culturalismo o absolutización de la crítica a la cultura preocupado por las identidades y subordinando las luchas socioeconómicas pendientes a discusiones casi exclusivamente centradas en la crítica cultural. El antiandrocentrismo se transforma cuando, con la incorporación masiva de la mujer al mundo del trabajo, se apuntala desde el discurso neoliberal que la mujer, para salir de la estructura opresiva, debe hacer prevalecer en sí misma lo mental y borrar o desdibujar lo que en ella es material, corpóreo. No obstante, como trabajadora asalariada y aportando otro salario al núcleo familiar, lo que esconde la «nueva narrativa del avance femenino y la justicia de género» (Fraser, 
2009: 98) ${ }^{3}$ es la realidad aumentada de la desigualdad y la injusticia (niveles salariales bajos, precariedad, descenso del nivel de vida, aumento de horarios laborales, etc.). Por su lado, el antiestatismo se resignifica a través de la aceptación de discursos en torno a un Estado liberado de responsabilidades, como la gestión de ayudas públicas o mecanismos de distribución social, transmitidas o cedidas a organismos como las ONG. Por último, el westfalianismo se troquela con la globalización y los discursos transnacionales. En resumen:

[...] Por una parte, el movimiento contracultural relativamente pequeño del periodo anterior se ha ampliado exponencialmente, difundiendo con éxito sus ideas por todo el planeta. Por otra, las ideas feministas han experimentado un sutil cambio de valencia en el contexto alterado. [...] Después de todo, este capitalismo prefería con creces afrontar las reivindicaciones de reconocimiento y no las reivindicaciones de redistribución, a medida que construye un nuevo régimen de acumulación sobre la piedra angular del trabajo asalariado de las mujeres, e intenta separar los mercados de una reglamentación social, para operar con la mayor libertad posible en una escala planetaria (Fraser, 2009: 101).

Al final de «El feminismo, el capitalismo y la astucia de la historia», con exceso de optimismo y esperanza, Fraser vislumbraba que, a partir de la elección en Estados Unidos del presidente Barack Obama, se podía dar el inicio del rechazo venidero del proyecto político del neoliberalismo (Fraser, 2009: 101). Tiempo después, desinflada la esperanza y desvelado el espejismo, la pensadora denominará a todo el periodo previo a la llegada a la presidencia de Donald Trump como el tiempo dominado por el bloque hegemónico del «neoliberalismo progresista» (Fraser, 2017). Para ella, el aparente oxímoron tras esta expresión no es tal pues, lo que en su momento planteara como posibilidad desasosegante, se materializó en la alianza de las corrientes liberales con los movimientos sociales (feminismo, antirracismo, multiculturalismo, ambientalismo y movimientos LGBTQ) con los sectores financieros y económicos estadounidenses, mezclando lo que parecería agua y aceite: las perspectivas políticas en torno a la distribución junto a las del reconocimiento. En sus palabras: «El bloque neoliberal-progresista combinó un programa económico expropiatorio y plutocrático con una política de reconocimiento liberalmeritocrática» (Fraser, 2017).

3 Los fenómenos de la resignificación en las narrativas sobre el avance femenino, en palabras de Fraser, se muestran con claridad en múltiples ámbitos como, por ejemplo, en el campo del liderazgo en las mujeres. Como han demostrado Reverter-Bañón y Medina-Vicent (2017), la conceptuación de la mujer-que-dirige se conjuga con la apelación al talento y la creatividad junto a remanentes del esencialismo de género anejo a la compartimentación de un conjunto de valores masculinos o femeninos. 
Habiendo repasado el proceso de reapropiación del neoliberalismo de objetivos planteados por los movimientos feministas, procede establecer la pregunta para investigar apropiadamente las medidas políticas japonesas. Así pues, ¡es el discurso de Abe acerca del womenomics un ejemplo más de esa reasignación de significado para nutrir las políticas neoliberales o se está ante un discurso, en esencia y aspiración, acorde con las luchas feministas en Japón y más allá de sus fronteras? Se tratará de interpretar qué sentido hay tras los discursos de Abe como el realizado en el 2014, en el Foro Económico Mundial, cuando exclamaba lo siguiente:

[...] Después de todo, la fuerza laboral femenina en Japón es la fuente menos utilizada. Japón se debe convertir en un lugar en el que las mujeres brillen. Para el 2020 conseguiremos que el $30 \%$ de las posiciones de liderazgo sean ocupadas por mujeres. Para tener a un mayor número de mujeres en puestos de liderazgo también se necesitará ayuda para las tareas de casa, el cuidado de los mayores y tareas similares. El PIB de Japón podría crecer un $16 \%$ más si las mujeres participaran en el mercado laboral tanto como los hombres. Esto es lo que me explicó Hilary Clinton. Me animó ampliamente (Abe, 2014).

\section{ORIGEN Y SIGNIFICACIÓN DEL CONCEPTO POLÍTICO- ECONÓMICO WOMENOMICS EN EL JAPÓN ACTUAL}

Como ya se comentó, el concepto womenomics propuso en 1999 la idea de «comprar la economía femenina» como salida para corregir el estancamiento y contracción del crecimiento económico. A pesar de los veinte años pasados desde que Matsui pusiera en circulación el planteamiento económico tras el womenomics», hoy se conecta con el llamado Abenomics.

En el mismo discurso del presidente Abe del 2014 se encuentra la definición de las tres flechas de las políticas económicas conocidas como Abenomics: adopción de políticas monetarias; política fiscal flexible y activar la inversión privada; y adopción de la filosofía tras el concepto womenomics en el apartado dedicado a exponer qué mecanismos deben servir para reiniciar (rebooting) el país entero (Abe, 2014).

Para reiniciar el país, Abe centra su atención en una de las reformas clave en cualquier Estado: la del mercado laboral. Así, en el discurso el presidente avanza las reformas que se han de emprender en el mercado laboral, pretendiendo dejar a un lado lo que denomina como «viejas industrias» para dar espacio a nuevas formas industriales con recursos humanos innovadores y creativos, en sus palabras. Y es ahí, en esa necesidad de recursos humanos in- 
novadores y creativos, el lugar exacto en el que se enlaza la mujer como la fuente de estos. Entre otras cosas, evitándose, de este modo, confrontar otra de las fuentes de recursos humanos habitual, a saber, la de trabajadores migrados de países terceros.

Si se vuelve ahora a la conceptualización que Matsui realiza sobre la cuestión en su actualización del 2014, «Womenomics 4.0: Predicar con el ejemplo», la política estratégica que el grupo de banca recomienda para Japón responde, en principio, a la defensa de lo que denominan con el término diversidad, es decir, a la equidad de representación entre géneros en el mercado laboral. $A$ priori, lo que se defiende es la integración efectiva e igual de la mujer japonesa en el mercado laboral, teniendo en cuenta que, según las estadísticas, en el 2014 la participación de la mujer en el mundo laboral es del 63\%, pero baja en comparación a otros países de la OCDE.

El diagnóstico que el equipo asiático de Goldman Sachs realiza apunta, por un lado, a los avances realizados desde el anterior informe (2010), constatando que, en efecto, se ha producido un ascenso de la población femenina integrada en el mercado laboral (del 6o \% al 63\%) pero que, no obstante, se debe incrementar hasta llegar a la equivalencia con los hombres activos laboralmente $(80,6 \%)$. ¿Cuáles son los motivos?

Primero: womenomics como instrumento para potenciar el crecimiento de dividendos. Siguiendo el planteamiento económico liberal del grupo bancario, los autores del informe constatan que el motivo de estancamiento en el país asiático se debe, en parte, al problema demográfico. Y el problema de envejecimiento y desequilibrio demográfico se puede corregir, precisamente, a través del aumento de la presencia de la mujer en el mundo laboral de un modo expandido. Así lo indican:

Si el nivel de empleo femenino del 2013 (62,5\%) ascendiera al nivel del masculino (80,6\%), este hecho añadiría 7,1 millones de empleados como fuerza de trabajo. Estimamos que el nivel absoluto del PIB de Japón subiría tanto como un 12,5\%. Siendo claros, no estamos argumentando que cada mujer japonesa debería trabajar fuera de casa. La decisión de trabajar fuera de casa o no es, obviamente, una elección individual y personal. Más bien, nuestro argumento es que a las mujeres japonesas que deseen trabajar fuera de casa (especialmente en jornada completa) no solamente se les debería dar la oportunidad, sino animarlas a hacerlo (Matsui, Suzuki, Tatebe y Akiba, 2014: 5-6).

Se trata, por tanto, de demostrar cómo se conecta el crecimiento económico (o de PIB) con el crecimiento de la tasa de mujeres en el mercado laboral. 
Segundo: womenomics y Abenomics. Como se ha ido indicando, el presidente Abe describió su intención de promover el trabajo femenino en Japón como una flecha adicional a la reforma del mercado laboral japonés. Así, según la agenda establecida por parte del primer ministro de cara al horizonte del 2020, se debían tener en cuenta los siguientes elementos de reforma laboral con directa implicación de la mujer: potenciar la presencia de mujeres en puestos de responsabilidad y liderazgo, al nivel del $30 \%$ para ese año; conseguir el $73 \%$ de participación en el mercado laboral de las mujeres en la franja de edad entre 25-44 años; subir el retorno al mercado de trabajo de las mujeres tras su maternidad del $38 \%$ al $55 \%$; reducir las listas de espera de los servicios y recursos para el cuidado infantil e incrementar el porcentaje de padres que se acogen a la baja por paternidad del $2,6 \%$ al $13 \%$.

Horizonte 2020 se visualiza como continuación de los cambios implementados previamente. El año de referencia es el 2010, cuando se afianzó un mayor número de mujeres en el mercado laboral, ampliando servicios de ayuda a la crianza de los niños y, a priori, tratando de introducir la diversidad de género en diversas áreas económicas. Pero el país sigue mostrando frenos a los pasos a dar para avanzar aún más en el sentido del concepto womenomics. Para empezar, a pesar del incremento, Japón sigue manteniendo un nivel de participación femenina en el mercado laboral bajo en relación con otros países de la OCDE. También se constata la falta de representación femenina en posiciones de relevancia, tanto en ámbitos públicos como privados: bajísima representación política de parlamentarias (77 mujeres de 722 representantes en las cámaras, baja y alta) o de puestos de liderazgo en empresas privadas. Se constata igualmente la brecha salarial (29\% de diferencia salarial) y desigualdad en las oportunidades laborales, así como un sistema de seguridad social e impuestos que imposibilitan la incorporación de las mujeres al mercado laboral. El cabeza de familia (marido) puede pedir una exención impositiva en concepto de tener a su cargo a su mujer siempre que ella no gane en torno a 10 ooo euros de salario anual. Por tanto, esto comporta que las mujeres se releguen, como mucho, a realizar trabajos en jornada parcial o a no trabajar, para mantener esa exención. Y, desde la visión empresarial, siguen en pie las reticencias de las compañías a implementar la diversidad de género en sus empresas (Matsui, Suzuki, Tatebe y Akiba, 2014: 11-16).

Así las cosas, para rebajar los problemas derivados de estos diferentes obstáculos, se pueden repasar las recomendaciones que realiza Goldman Sachs. Al Gobierno se le propone liberalizar los sectores dedicados al cuidado infantil; reformar la migración para potenciar la entrada de trabajadores que realicen 
tareas de cuidadores; revisar el sistema impositivo; modificar los sistemas de beneficios por cuidado infantil para condicionarlos a la participación de la mujer en el mundo laboral, así como potenciar también la baja por paternidad; animar a la diversidad de género en las empresas; adoptar el modelo holandés de media jornada pero con trabajo igual; potenciar la participación de la mujer en los espacios públicos políticos; promover a la mujer como emprendedora y facilitar la reentrada de la mujer al mundo laboral con formación y centros de ocupación. Al sector privado, se le recomienda potenciar la diversidad; crear ambientes de trabajo más flexibles; adoptar esquemas justos y objetivos sobre evaluación, compensación y promoción; establecer objetivos de diversidad de género claros y medibles e introducir contratos de empleo más flexibles. A la sociedad, se le recomienda batallar por desmontar mitos y animar a una mayor igualdad en las tareas domésticas (Matsui, Suzuki, Tatebe y Akiba, 2014: 17).

Detrás de todas las recomendaciones, la lógica económica es fácilmente reconocible. Constatado el problema esencial (demográfico) y que Japón sufre de falta de mano de obra, las mujeres, muchas de ellas con una formación académica alta, podrían suplir esas carencias. Y ello potenciaría el consumo interno y crearía, a su vez, el surgimiento de nuevas demandas en áreas como las comprendidas en el sector servicios.

Esa misma lógica económica detrás de la conceptuación de womenomics en el caso japonés y el éxito internacional del planteamiento se constata mirando la repercusión de la idea en foros muy influyentes para el pensamiento político y económico. En el artículo publicado en el 2006 titulado «Mujeres y la economía mundial. Una guía de womenomics», The Economist, replicando casi punto por punto las claves de Matsui desde Goldman Sachs, amplifica la cuestión más allá de las fronteras japonesas. Para la revista, «las mujeres son ahora el motor (engine) más poderoso del crecimiento global» (The Economist, 2006). Empleando una de las respuestas aportadas por Richard B. Freeman, uno de los primeros economistas que a finales de la década de 1990 ya analizaba el fenómeno de la feminización del trabajo en el contexto estadounidense, ante la pregunta sobre los motivos del patrón y la extensión hacia otros países (Freeman, 2000: 3-22), The Economist responde de igual modo al señalar una de las causas que puede sintetizarse en el cambio acaecido en el mundo laboral y productivo. Es decir, la transformación del mercado laboral desde la previa composición primordial de los sectores industriales hacia el sector servicios, en el cual las mujeres habían desempeñado sus tareas habitualmente. 
Según la revista, el incremento del trabajo femenino en los países desarrollados se ha visto ayudado por la expansión del sector servicios y el declinar del trabajo industrial o productivo en la manufactura. Caso distinto, no obstante, como no se le olvida señalar a la publicación, es que gran parte de las economías emergentes en el este asiático, como en el caso de China, no podrían explicar ni su dinamismo ni el crecimiento de las exportaciones en sectores como el textil o el sector dedicado a la manufactura de ropa sin la realización del trabajo (6o-8o \% de la ocupación) en manos de las mujeres. Simplemente se está constatando un hecho que nunca se debe olvidar cuando se repiten discursos sobre los milagros económicos de los últimos 25 años.

Asimismo, la publicación se hace eco de la mencionada minusvaloración del trabajo no asalariado en casa. Traducido a nivel monetario, haría recaer en las espaldas de las mujeres más de la mitad de la producción del PIB en las economías desarrolladas. Conforme a The Economist:

Sin embargo, la mayoría de las mujeres trabajadoras (= asalariadas) todavía son responsables de la carga de las tareas en sus hogares. En las economías desarrolladas, las mujeres producen en torno al $40 \%$ del PIB oficial. Pero si se añadiera el trabajo en casa [aplicando valor de las horas trabajadas en el promedio de los niveles salariales de personas (contratadas) para la ayuda en el hogar o en tareas de cuidado de los hijos], entonces las mujeres probablemente producirían algo más de la mitad del total (The Economist, 2006).

Por otro lado, no solamente es esencial tener en cuenta estas variables correlacionadas con el PIB de los países, sino el otro elemento de dinamismo al que se alude en el artículo al referir al "poder de las chicas» (Girl power): las mujeres no solamente introducen dinamismo dentro del mercado global desempeñando tareas directamente relacionadas con sus puestos de trabajo asalariado (y no asalariado). Son también un motor del dinamismo económico, como consumidoras, emprendedoras, managers e inversoras. En particular, el foco se pone en el rol desempeñado tradicionalmente por las mujeres como administradoras del presupuesto en los hogares, por tanto, responsables de las decisiones de compra como consumidoras: dice el artículo, responsables de decidir respecto a servicios de salud o inmobiliarios hasta muebles o comida (The Economist, 2006).

Otro aspecto que apuntala más cuál es la lógica económica frente a la lógica de igualdad y justicia y, además, incluye tintes deterministas respecto a la supuesta diferencia de carácter entre mujeres y hombres, es el siguiente: 
Promover un mejor uso (use) de las competencias femeninas no es simplemente un tema de justicia. Muchos estudios sugieren que también es bueno para los negocios. [...] en compañías americanas con más mujeres en puestos de dirección comporta un mayor retorno en equidad que aquellas con menos mujeres en la cúspide. Esto puede deberse al hecho de que equipos mezclados de hombres y mujeres son mejores resolviendo problemas o discerniendo riesgos externos que aquellos solamente compuestos por un único sexo. Estudios sugieren también que las mujeres son habitualmente mejores que los hombres formando equipos y comunicando (The Economist, 2006).

Complementariamente, replicando el argumento de Matsui que Abe retorna en su discurso del año 2014 en el Foro Económico Mundial, The Economist constata lo que a su juicio es la mujer como fuente infrautilizada, en Japón, con cifras similares a las antes comentadas, pero, llevando el argumento al continente europeo, con una misma infrautilización, a su juicio, en países como Italia, Alemania o Francia. Aquí el parámetro respecto al segmento de edad es importante y para tener en cuenta conectado con el argumento de la baja tasa de natalidad y la debacle demográfica de una Europa envejecida:

Según Kevin Daly, de Goldman Sachs, entre las mujeres con edad comprendida entre 2529 años, la participación [laboral] en la UE (proporción de mujeres que están trabajando o buscando trabajo) es la misma que en América [65\%]. Entre las mujeres de 55 a 59 años, es del $50 \%$ mientras que en América es del $66 \%$. Con el tiempo, el trabajo femenino en Europa seguramente crecerá, para beneficio de sus economías (The Economist, 2006)

Por último, se debe hacer una mención especial a uno de los argumentos más claramente economicistas, pero, además, impulsados por el determinismo naturalizante del ser-hacer como mujer. Se trata de correlacionar dos variables: formación y educación de las mujeres con mayor productividad y mayor beneficio en su tarea como educadoras:

En particular, hay una fuerte evidencia respecto al hecho de que la educación de las chicas contribuye a la prosperidad. Probablemente es la mejor inversión que se puede hacer en el mundo desarrollado. Las mujeres mejor educadas no solamente son más productivas, sino que crían a niños más saludables y mejor educados (The Economist, 2006)

La lógica o argumento económico es, como se ha visto, evidente. Y no se habla de un contexto privativo del caso japonés, sino de la sugerencia y recomendación para otros contextos, por supuesto, en las economías en vías de desarrollo o emergentes, así como una receta válida para la recesiva economía europea. 


\section{ABE-WOMENOMICS: ¿GIRO HACIA LA EQUIDAD GENUINA O HACIA EL PATRÓN PRODUCTIVISTA?}

El análisis realizado aspira a dar respuesta a la pregunta formulada en su momento: ¿Es el Abe-Womenomics un ejemplo de un reasignado feminismo al servicio de las políticas neoliberales o se está ante un discurso acorde con las luchas feministas en Japón y más allá de sus fronteras?

Solamente teniendo en cuenta el informe estratégico de Goldman Sachs, la respuesta parecería bastante clara. El diagnóstico y las recomendaciones que realiza el grupo bancario se presentan en clave puramente económica. Esto incluso en el apartado referido a aspectos que buscan incidir en la sociedad y la igualdad en el ámbito doméstico. Sucede por un motivo bien sencillo: para reintroducir y ampliar la fuerza de trabajo femenina en el mercado laboral japonés, se trata de destruir todos los obstáculos. Y uno de ellos, indudablemente, refiere a una sociedad que muestra reticencias enraizadas en valores en los que la mujer sigue estando subordinada a mecanismos fuertemente patriarcales.

Agudizar la mirada crítica pasa por ver el motivo último para que la estrategia económica preconizada con la nomenclatura womenomics incluya entre sus recomendaciones la necesidad de desmontar falsos mitos sobre el papel de la mujer en el mundo del trabajo. Los mitos que señala el informe de Goldman Sachs se deben leer desde este prisma crítico.

En primer lugar, habla del mito construido sobre la idea de que las mujeres japonesas dejan de trabajar a causa de los factores de atracción o incitadores (pull factors), ${ }^{4}$ siendo tales factores que las mujeres abandonan el mundo laboral a causa de su trabajo en el hogar al cuidado de los niños y los ancianos. En este caso, se dice que es una falsa idea porque los datos apuntan a que el principal motivo de abandono del mundo laboral se produce más bien por la insatisfacción de las mujeres en sus puestos de trabajo y en su desarrollo profesional y por la falta de horizonte de crecimiento profesional en sus carreras.

La segunda preconcepción consiste en afirmar que las mujeres no desean volver al mundo laboral tras su maternidad. En este caso, los datos aportados también lo desmienten porque, a pesar de que las mujeres desearían volver al mercado laboral, tras la maternidad se encuentran con pocas oportunidades

4 En el lenguaje económico, se habla de push and pull factors, esto es, factores incitadores o disuasivos, en este caso, de la integración de la mujer en el mercado laboral. 
para cumplir con su voluntad. Otra variable es la falsa creencia que afirma que, a mayor número de mujeres trabajando, aumenta la disminución de trabajos para los hombres. El modo economicista de acabar con esta idea es simple: Japón tiene necesidad de fuerza laboral. Actualmente, lejos de estar en una situación en la cual la tasa de paro fuera amplia (menos del $5 \%$ frente al 15$20 \%$ de España) (IMF, 2018) más bien la incertidumbre se produce por una próxima falta de mano de obra. Y, por último, la creencia que establece que, a mayor participación femenina en el mundo laboral, mayor descenso de nacimientos. En este caso, se intenta desmontar aludiendo a que dicha correlación entre desarrollo profesional femenino y reducción de las tasas de maternidad no está empíricamente demostrada (Matsui, Suzuki, Tatebe y Akiba, 2014: 2529).

Si se lee la descripción de estas barreras sociales en la clave crítica indica$\mathrm{da}$, en vez de considerarse que el planteamiento apunta hacia un genuino movimiento en pro de la justicia y la equidad de la mujer y el hombre en la sociedad japonesa actual, lo que aparece como sospecha es una instrumentalización de esa equidad para servir a unos fines, sin lugar a duda, económicos. Como comenta Hiroko Takeda:

En suma, la discusión sobre womenomics convierte en un caso de «negocio» la promoción de la participación laboral de las mujeres en Japón en la forma del lenguaje estratégico de un banco de inversión. Para proveer a los clientes la información necesaria para decidir realizar inversiones, los informes sobre womenomics identifican la infrautilización de la fuerza laboral femenina como un obstáculo estructural que impide a la economía japonesa sostener y estimular el desarrollo económico y hacer una llamada determinante para el cambio (Takeda, 2015: 6).

Si se habla de un caso de negocio, cabe la posibilidad de ver en la estrategia reformista defendida la vieja estrategia del liberalismo-nacionalista. Si la razón que marca la agenda política detrás de la Abe-Womenomics es esencialmente económico-productiva, se está ante la reactualización de ese principio paritario que, por ejemplo, tiene larga historia en las diferentes revoluciones industriales, por la cual, a mayor población productiva, menor población que solamente consuma recursos. No se defienden la igualdad y la justicia entre géneros como valores defendibles por sí mismos, sino instrumentalizados como medio efectivo para otro valor superior: el crecimiento económico.

Es el llamado «argumento económico» (Takeda, 2015: 6) el que está detrás del cambio de eje, del viraje, de un gobierno, el de Abe, y de un partido que, precisamente, se había significado por defender durante décadas la protección 
de los valores culturales tradicionales. Es decir, protección de esa esencialidad prescrita para las mujeres que encaja con la idea de proteger la ecología de la cultura japonesa, según el concepto de la pensadora ligada al Partido Liberal Democrático (PLD) de Abe y amiga de este, Michiko Hasegawa. Ella advertía de los peligros de avanzar en el camino de las legislaciones de equiparación de los derechos y las oportunidades laborales a partir de los años 1990, considerando que, si se regulaba para superar la segregación laboral y profesional entre hombres y mujeres, no se hacía sino destruir ese medioambiente tradicional: el de una feminidad enlazada, estática y esencialmente, a ser ama de casa, madre y educadora (Ueno, 2005: 225-245). Por tanto, conviene considerar que, tras el cambio de dirección del gobierno Abe, está, precisamente, ese argumento económico:

Es decir, la promoción de la igualdad de género está puesta como un instrumento útil para afrontar, primero, la desaceleración de la economía en el período post-burbuja, segundo, hacer frente a las presiones de la globalización económica, tercero, enfrentarse a los rápidos cambios demográficos y de modos de vida, $\mathrm{y}$, al hacerlo, racionalizar y mejorar la economía japonesa, ayudando a que vuelva a la senda de crecimiento (Takeda, 2015: 7).

Ahora se puede revisar si es el caso que diversas de las aspiraciones de los movimientos feministas de los años 1970, también representados en Japón, se resignificaron para apoyar las nuevas propuestas económicas del neoliberalismo.

Por un lado, la lucha de los diferentes colectivos feministas japoneses de la década de 1970 consiguió una normativa que, a priori, debía servir de base para aposentar jurídicamente la igualdad de oportunidades. La ley marco por la igualdad de oportunidades se aprobó en 1985, siendo efectiva a partir de 1986. El problema con ese marco normativo es, en palabras de Mackie, que simplemente se trataba de una farsa-de-ley (travesty) o, dicho de otro modo, de una ley puramente formal pero no sustantiva. Esto porque, entre otras cosas, no regulaba acciones punitivas para corregir todas aquellas acciones desiguales para las mujeres respecto a su presencia en el mundo laboral:

La ley está basada en una filosofía de la «igualdad de oportunidades» más bien que en «igualdad de resultados» y no está apoyada con programas de acción afirmativa [discriminación positiva]. Las compañías serán «animadas» a «hacer esfuerzos» para abolir la discriminación en la contratación, despido, transferencia y promoción. Se les prohíbe discriminar en relación con la formación, asistencia pública, jubilación y reducción [fiscal], aunque no se indican multas [en el caso de incumplimiento] (Mackie, 1988: 65). 
Por otro lado, es posible ver que, en el actual planteamiento del AbeWomenomics, se han sabido emplear discursos e ideas propulsadas por los movimientos feministas a partir de la década de 1980 para legitimar la sociedad capitalista en su actual estructuración de economía extractiva. Las implicaciones que se esconden tras algunas de las estrategias preconizadas a partir del argumento económico se sustentan en deducciones que merecen leerse con atención. No procede dejarse llevar por la superficie de un discurso que, en apariencia, empleando las mismas nociones y conceptos, no apunta hacia las mismas metas y objetivos de los movimientos feministas.

Se puede atender a la crítica que se realiza a este tipo de iniciativas que ponen como bandera del cambio el potenciar a segmentos bien delimitados entre la población femenina. Como comenta Szendi Schieder, la estrategia apunta hacia la paridad estrictamente referida a los sectores femeninos dentro de la clase social más elitista (mujeres con amplia formación académica que podrían desempeñar cargos de relevancia pública y privada) y conviene con la idea de romper con el techo de cristal. El mensaje puede funcionar como opiáceo frente a la base social de las mujeres de clase media o baja que siguen sosteniendo la pirámide de un sistema laboral en el que la norma es la desigualdad y la precarización laboral (Szendi Schieder, 2014: 55-56). La frase que sintetiza esta perversión del lenguaje igualitario es de Laurie Penny: «no os preocupéis por el techo de cristal: el sótano está inundado» (Penny, 2001, en Szendi Schieder, 2014: 56). Lo que parece esconderse tras el discurso feminista con el que se proyecta la estrategia Abe-Womenomics no es sino la instrumentalización de esa supuesta liberación prescrita desde arriba (la élite gubernamental y económica) para favorecer la mercantilización neoliberal de la mujer como fuerza de trabajo (Szendi Schieder, 2014: 56-57).

Además, esta mercantilización de la mujer como fuerza de trabajo no es un fenómeno nuevo en el país. Más bien lo contrario. A pesar de que, en su discurso del 2014, Abe habla específicamente de algo así como una historia de infrautilización de la fuerza laboral femenina en Japón, la historia moderna del país no se entiende a partir de Meiji (1868-1912) sin tener en cuenta el papel de las mujeres trabajadoras en el ciclo de la revolución industrial de finales del siglo XIX. Fueron las mujeres las que desempeñaron tareas de importancia fundamental para la industria, fundamentalmente textil, de fines del siglo XIX y principios del Xx. Jóvenes de clase baja o provenientes de las zonas rurales formaban parte de las plantas textiles y otras fábricas, motor de la industrialización (Hane, 1988: 13). Como dato adicional, no conviene olvidar que, a pesar de la limitación del ejercicio político de las mujeres en aquella época, algunas 
se organizaron en fecha temprana (1885) para reclamar sus lícitos derechos laborales en forma de huelgas en las industrias textiles. Se desmonta con ello ese sofisma alrededor de la infrautilización de la mujer en el mercado laboral. Siguiendo a Szendi Schieder, más que hablar de fuente infrautilizada, sería posible describir la historia moderna de la mujer en el mundo laboral en el país asiático como la de una fuente explotada (Szendi Schieder, 2014: 57).

Si se prosigue aún más con la crítica a esta idea de la mujer como fuente laboral infrautilizada, es conveniente añadir alguna otra cuestión. Al hablar de fuente infrautilizada, solamente se considera como trabajo todo aquel que se corresponde con una remuneración salarial. Se deja a un lado, en Japón como en nuestro contexto social próximo, todas aquellas áreas no remuneradas relativas al cuidado de la familia en sentido amplio. Al no simbolizarse en el lenguaje monetario del salario, se olvida que corresponden con un coste monetario que, de otro modo, debería estar sustentado por un mejor y más fuerte sistema de protección y seguridad social que colaborara haciendo frente al cuidado de los hijos y de las hijas, los mayores, la organización y la administración del hogar, etc. Y no se trata solamente de aquellas mujeres que abandonan la posibilidad de desarrollar su carrera y se centran en el hogar. Se trata también de aquellas mujeres que, contratadas en puestos de trabajo a tiempo parcial y en situaciones de precarización, cargan con el patrón habitual en gran parte de las sociedades (más o menos avanzadas económica y socialmente) en las que sigue siendo tarea difícil romper con la llamada doble carga: como trabajadoras asalariadas precarizadas y al frente de las tareas domésticas y de cuidados.

Curiosamente, ese hecho de la doble carga no pasó por alto para el planteamiento del gobierno japonés actual. Todo lo contrario, pero para transmitir esa cadena de precarización y de minusvaloración del trabajo doméstico. Se puede recordar la idea expresada por Abe: «[...] Para tener a un mayor número de mujeres en puestos de liderazgo también se necesitará ayuda para las tareas de casa, el cuidado de los mayores y tareas similares» (Abe, 2014). La significación del argumento es clara: para que el segmento femenino perteneciente a la élite social pueda llegar a puestos de liderazgo (romper el techo de cristal), su trabajo en las tareas domésticas debe ser realizado por algún colectivo subordinado. Se piensa en (mujeres) migrantes cuya entrada en el país debe servir para cumplir con funciones de servir como trabajadores domésticos. Dice Mackie: 
Otra política controvertida anima a la utilización de migrantes para realizar trabajos domésticos en las zonas económicas especialmente designadas a fin de mejorar la participación laboral de las mujeres. Esta política se ha criticado por reforzar la idea de que las tareas domésticas y el cuidado de los hijos son responsabilidades femeninas. Deja intacta una cultura del trabajo que hace difícil para los trabajadores a jornada completa participar en el trabajo de cuidado y doméstico y conseguir el equilibrio entre la vida personal y laboral (Mackie, 2016).

Se está, nuevamente, ante la clásica instrumentalización de un colectivo social para beneficio de otro: en este caso, de migrantes cuya entrada será aceptada en el país para servir a un área laboral muy determinada para beneficio de un segmento pequeño de mujeres, aquellas que ya parten de una ventaja económica, formativa, etc.

En el esquema de sociedades que forman parte del capitalismo avanzado, el desarrollo individual y el de la sociedad de libre mercado se sustenta sobre el ser productivos, eficientes y autosuficientes. Una ética de la productividad que se impone sobre la ética de la justicia e igualdad sociales.

\section{CONSIDERACIONES FINALES}

En ocasiones, el uso del concepto neoliberalismo se iguala, con pocos matices, con otros elementos propios de la filosofía política liberal. Pero a lo que hace referencia política y económicamente es a una serie de medidas ampliamente extendidas en el mundo capitalista: desregulación del comercio y las finanzas; privatización de servicios antes garantizados por el Estado; desregulación activa de condiciones macroeconómicas por parte del Estado, en particular, del empleo; reducción de gasto social; reducción de impuestos aplicados a empresas y familias; desplazamiento del poder a favor del capital y debilitación de la capacidad de negociación colectiva de los trabajadores; temporalidad laboral; competitividad empresarial desenfrenada e introducción de principios de libre mercado en las remuneraciones laborales (Garzón Espinosa, 2010). El planteamiento detallado de las diferentes normativas que encajan con la implantación de womenomics en Japón tiene que ver con, por ejemplo, el primer punto: la desregulación. Sin ir más lejos, Abe propuso implementar la estrategia bien conocida en China: las zonas económicas especiales (ZEE) 
(González, 2014).5 Las ZEE probarán modelos de desregulación que, evidentemente, tendrán incidencia en el mundo laboral. Señala Takeda:

Lo que ilumina la fuerte orientación del gobierno Abe hacia la desregulación laboral más que nada es, sin embargo, un esquema de la zona especial de empleo en el que las firmas privadas pueden virtualmente tener las manos libres en relación con la dirección de las relaciones industriales. El esquema es uno de los centros de la estrategia de crecimiento de Abenomics, junto a la promoción de la fuerza laboral femenina, y, en su forma actual, las firmas privadas solamente necesitan situar sus oficinas centrales en el interior de la zona especial para ejercer ese privilegio (Uchihashi, 2015). En otras palabras, las revisiones en curso de las regulaciones laborales se dirigen hacia mayor desregulación que va a comportar que los trabajos irregulares permanezcan en esa posición e incrementar las horas de trabajo para los trabajadores del sexo masculino (Takeda, 2015: 15).

Esta potenciación de la desregulación económica y de la normativa laboral tiene, claro, su incidencia en el caso de las mujeres. Así, teniendo en cuenta los datos y los resultados previos de otros procesos de desregulación implementados, para el segmento de trabajadoras comportará que el colectivo amplio de ellas (no de aquellas que pertenecen a la clase social más aventajada) permanezca en condiciones de precariedad laboral, precintadas en trabajos a media jornada. A nadie se le escapa que la feminización de los contratos sin continuidad, fragmentarios, temporales y a media jornada, mayoritariamente en manos de las mujeres, agudiza el empobrecimiento. La pobreza en Japón y en otras sociedades que siguen el patrón neoliberal tiene nombre de mujer. Y seguirá manteniéndolo a partir de la desregulación laboral y la rebaja en la garantía de derechos laborales antes protegidos por la estructura estatal y jurídica.

El título del artículo de Takeda reza así: «Todos los deseos del Estado de Japón son mujeres brillantes (y sus familias): Tatemae y Honne de AbeWomenomics». Con Fraser se vio la idea de la resignificación, es decir, atender a los procesos de adquisición de un nuevo sentido diferente al originario de aspiraciones y metas de la segunda ola feminista de los años 1970 y el mecanismo de acomodación de esas aspiraciones, metas e incluso su lenguaje emprendido

5 Según declaraciones del exministro Takenaka recogidas hace unos años en el diario El País: «Las zonas económicas especiales van a aplicar un modelo económico muy desregulado a modo de prueba y quizá luego se pueda aplicar a toda la economía. Es muy similar al sistema que China aplica en el área de Shanghái o en Shenzhen [...] La estrategia de crecimiento es muy importante, la economía japonesa está muy regulada y hay muchos intereses creados que lo hacen muy difícil. Hay que impulsar un proceso de desregulación y privatización, pero para ello es necesario un liderazgo político muy fuerte» (González, 2014). 
por el neoliberalismo. El tatemae y el honne apuntan a denunciar el mismo proceso de resignificación.

La retórica de ciertos discursos tiene impacto simbólico y mediático. Esto sucede, por ejemplo, al leer el discurso realizado por Abe ante la Asamblea General de las Naciones Unidas en el 2013 en el que declaró la intención de crear «una sociedad en la que las mujeres brillen» (Abe, 2013). Pero toda luz necesita de la sombra. Es decir, la asimetría entre trabajadores y trabajadoras, clases sociales, etc., se esconde en la proyección de luz retórica que deja sin atender los procesos contemporáneos de empobrecimiento femenino y la reactualización de discursos viejos empleados para instrumentalizar a la mujer al servicio del argumento económico.

Habría mucho más que discutir sobre los diversos procesos de resignificación. Lo que ha clarificado el caso específico japonés analizado es confirmar el proceso binario político: entre la proyección hacia afuera (tatemae-建前) de lo que significa womenomics y la verdadera intención, en el fondo de sí (honne本音). Entre lo público y lo privado.

Son muchos los interrogantes abiertos tras conocer los pros y los contras de womenomics, los aspectos que se muestran y se ocultan, la mantenida precarización del trabajo femenino, etc. Con todo, una cuestión es central: conviene preguntarse si el país puede permitirse confiar exclusivamente en womenomics como la solución ante un futuro incierto en un contexto cambiante como el actual. Es decir, si no es el caso que, además de esta estrategia de reintegración de la mujer en el mundo laboral, no tendrá que hacer frente a otras reformas pospuestas: por ejemplo, ampliar las posibilidades de entrada de migrantes o buscar otros mecanismos para incrementar las tasas de natalidad.

\section{BIBLIOGRAFÍA}

Abe, Shinzō (26 de septiembre, 2013). Address by Prime Minister Shinzo Abe at The Sixty-Eighth Session of The General Assembly of The United Nations. Recuperado de: http://japan.kantei.go.jp/g6_abe/statement/2013og/26generaldebat e_e.html [Consultado el 14 de febrero de 2017]

Abe, Shinzō (22 de enero, 2014). A New Vision from a New Japan. The World Economic Forum 2014 Annual Meeting. Recuperado de: 
http://japan.kantei.go.jp/96_abe/statement/201401/22speech_e.htm 1 [Consultado el 27 de enero de 2017]

Butler, Judith (1990). Gender Trouble: Feminism and the Subversion of Identity. London: Routledge.

Fraser, Nancy (2009). El feminismo, el capitalismo y la astucia de la historia. New Left Review, 56, 87-104. Recuperado de: http://newleftreview.es/authors/nancy-fraser [Consultado el 30 de julio de 2018]

Fraser, Nancy (2017). From Progressive Neoliberalism to Trump-and Beyond. American Affairs, I, 4, 46-64. Recuperado de: https://americanaffairsjournal.org/2017/11/progressiveneoliberalism-trump-beyond/ [Consultado el zo de enero de 2018] Freeman, Richard B. (2000). The Feminization of Work in the USA: A New Era for (Man)kind? En Gustafsson, Siv S. y Meulders, Danièle E. (Eds.). Gender and the Labour Market. Econometric Evidence of Obstacles to Achieving Gender Equality (3-22). New York: Macmillan-St. Martin's Press.

Garzón Espinosa, Alberto (19 de octubre, 2010). Neoliberalismo, características y efectos. Economía Crítica y Crítica de la Economía. Recuperado de: $\quad$ http://www.economiacritica.net/?p=15 [Consultado el 14 de febrero de 2017]

González, Alicia (13 de junio, 2014). Japón prepara otra oleada de reformas. El gobierno bajará el tipo sobre sociedades e impulsará zonas económicas especiales. El País. Recuperado de: http://economia.elpais.com/economia/2014/o6/13/actualidad/1402 684146_784139.html\#comentarios [Consultado el to de febrero de 2017

Hane, Mikiso (1988). Reflections on the Way to the Gallows. Rebel Women in Prewar Japan. Berkeley, Los Angeles, London: University of California Press.

IMF (2018). World Economic Outlook (WEO). Washington: International Monetary Fund. Recuperado de: 
http://www.imf.org/external/datamapper/LUR@WEO/OEMDC/ ADVEC/WEOWORLD [Consultado el 31 de julio de 2018]

Mackie, Vera (1988). Feminist politics in Japan. New Left Review, I/167, $53-76$.

Mackie, Vera (1 de agosto, 2016). Closing the gender gap in Japan East Asia Forum -Economics, Politics and Public Policy in East Asia and the Pacific. Recuperado de: http://www.eastasiaforum.org/2016/o8/o1/closing-the-gender-gapin-japan/ [Consultado el 27 de enero de 2017]

Matsui, Kathy, Suzuki, Hiromi, Tatebe, Kaazunori y Akiba, Tsumugi (3o de mayo, 2014). Womenomics 4.o: Time to Walk the Talk. Japan: Portfolio Strategy. US: The Goldman Sachs Group Inc. Recuperado de: https://www.goldmansachs.com/ourthinking/pages/macroeconomic-insights-folder/womenomics4folder/womenomics4-time-to-walk-the-talk.pdf [Consultado el 30 de julio de 2018]

Reverter-Bañón, Sonia y Medina-Vicent, Maria (2017). Intersecciones entre liderazgo y feminismo. Dossiers Feministes, 22, 5-12. doi: 10.6035/Dossiers.2017.22.1

Szendi Schieder, Chelsea (2014). Womenomics vs. Women: Neoliberal Cooptation of Feminism in Japan. Meiji Journal of Political Science and Economics, 3, 53-60. Recuperado de: http://mjpse.meiji.jp/articles/files/03-05/03-05.pdf [Consultado el 30 de julio de 2018]

Takeda, Hiroko (2015). All the Japan State Wants is Shining Women (and Their Families): Tatemae and Honne of Abe-Womenomics (Draft). Recuperado de: https://www.psa.ac.uk/sites/default/files/conference/papers/2015/ Abe-Womenomics_R_o.pdf [Consultado el zo de enero de 2017] Ueno, Chizuko (2005). In the Feminine guise: A trap of reverse orientalism. En Calichman, Richard F. (Ed.). Contemporary Japanese Thought (225-245). New York: Columbia University Press. 
Women and the world economy. A guide to womenomics. The future of the world economy lies increasingly in female hand (12 abril, 2006). The Economist. Recuperado de: http://www.economist.com/node/6802551 [Consultado el 26 de enero de 2017] 
86 RECERCA · DOI: http://dx.doi.org/10.6035/Recerca.2019.24.2.4 · ISSN: 1130-6149-pp. $63-86$ 